\title{
Experimental Study of Flow around a Circular Cylinder inside a Bubble Plume
}

\author{
Tomomi Uchiyama1, Yukihiro Ishiguro² \\ ${ }^{1}$ Institute of Materials and Systems for Sustainability, Nagoya University Furo-cho, Nagoya, Japan \\ ${ }^{2}$ Graduate School of Information Science, Nagoya University Furo-cho, Nagoya, Japan \\ Email: uchiyama@is.nagoya-u.ac.jp
}

Received 18 March 2016; accepted 11 July 2016; published 14 July 2016

Copyright (C) 2016 by authors and Scientific Research Publishing Inc.

This work is licensed under the Creative Commons Attribution International License (CC BY).

http://creativecommons.org/licenses/by/4.0/

(c) (i) Open Access

\section{Abstract}

This study experimentally explores the flow around a cylinder with circular cross-section placed inside a bubble plume. Small gas bubbles with diameter smaller than $0.06 \mathrm{~mm}$ are released from electrodes on the bottom of a water tank by electrolysis of water. The bubbles induce water flow around them as they rise because of buoyancy. Inside the generated bubble plume, a cylinder with diameter $D$ of $30 \mathrm{~mm}$ is placed at 6.5D above the electrodes. The bubbles and water flow around the cylinder are visualized, and the bubble velocity distribution is measured. The experiments elucidate the bubble behavior around the cylinder, the separated shear layers originating at the cylinder surface, their roll-up, the bubble entrainment in the resultant large-scale eddies behind the cylinder, and the vortex shedding from the cylinder.

\section{Keywords}

Bubble Plume, Separated Shear Layer, Cylinder Wake, Flow Visualization, PIV

\section{Introduction}

When gas bubbles are successively released into liquid, they rise because of buoyancy with inducing the liquid flow around them. Such bubble-driven flows or bubble plumes are used in various engineering processes that involve matter and heat transfer, mixing, and chemical reactions. Several studies have examined bubble plumes and methods to predict the entrained liquid flow rate [1] [2] and plume characteristics [3] [4]. The effect of bubble flow rate on the meandering motion of rising bubbles has also been investigated [5]. Moreover, methods to simulate bubble plumes have been presented [6]-[10], and they have successfully grasped the bubble movement and the induced eddies having various scales.

In engineering devices handling bubble plumes, interactions between device members and bubble plumes are frequently observed. The author [11] numerically simulated a bubble plume across a staggered tube bundle to 
investigate the bubble distribution and the unsteady force acting on the tubes. Murai et al. [12] visualized bubble plumes around cylinders with circular and triangular cross-sections and clarified the formation of a large-scale wake with no bubbles behind the cylinders. The bubble diameter ranged from 3 to $5 \mathrm{~mm}$, and therefore the ratio of the diameter to the representative length of the cylinders $(50 \mathrm{~mm})$ was relatively large. Because the characteristics of bubble plumes depend on the bubble terminal velocity or bubble diameter, bubble plumes around a solid body are also affected by the bubble diameter. However, there are no studies on flows around a solid body in a bubble plume consisting of small bubbles with diameter smaller than $0.1 \mathrm{~mm}$. Understanding such flows is very important to build guidelines for designing devices handling plumes produced by micro-bubbles.

This study experimentally investigates the flow around a cylinder with circular cross-section in bubble plumes induced by small bubbles. The experiment is performed in a water tank. Small hydrogen and oxygen bubbles are generated by electrolysis of water at the bottom of the tank. The mean bubble diameter is about $0.05 \mathrm{~mm}$. The bubbles rise owing to buoyancy and induce water flow around them. The bubbles and water flow around the circular cylinder are visualized, and the bubble velocity distribution is measured. The experiments successfully clarify the bubble movement, the separated shear layers originating at the cylinder surface, the entrainment of bubbles in large-scale eddies, and the vortex shedding from the cylinder.

\section{Experiment}

\subsection{Experimental Setup}

Figure 1 shows a schematic of the experimental setup. The water tank is made of transparent acrylic resin to enable flow visualization. The width and depth of the tank are both $14.7 D$, and the height is $19.7 D$. The water level is $16.7 D$. The top of the tank is open to the atmosphere. A cylinder with diameter $D$ of $30 \mathrm{~mm}$ is horizontally placed in the tank, and five carbon rods are placed at the bottom of the tank. The rod diameter is $0.16 D$. The origin of the coordinates is at the center of the carbon rods. The $x-y$ plane is horizontal and the $z$-axis is vertical.

Figure 2 shows the carbon rods and their positions relative to the cylinder. The length of each rod is $3.3 D$. The rods are arranged at intervals of $0.7 D$ along the $x$-axis on the tank bottom. Two rods are used as anodes, and the others are used as cathodes. Applying a DC voltage between the electrodes generates small oxygen and hydrogen bubbles at the anodes and cathodes, respectively. The generated bubbles rise in the water because of buoyancy with inducing water flow around them. Inside the generated bubble plume, a circular cylinder is horizontally placed. The cylinder length is $6.5 \mathrm{D}$ and its axis is orthogonal to the carbon rods. Its distance from the tank bottom is $6.5 \mathrm{D}$.

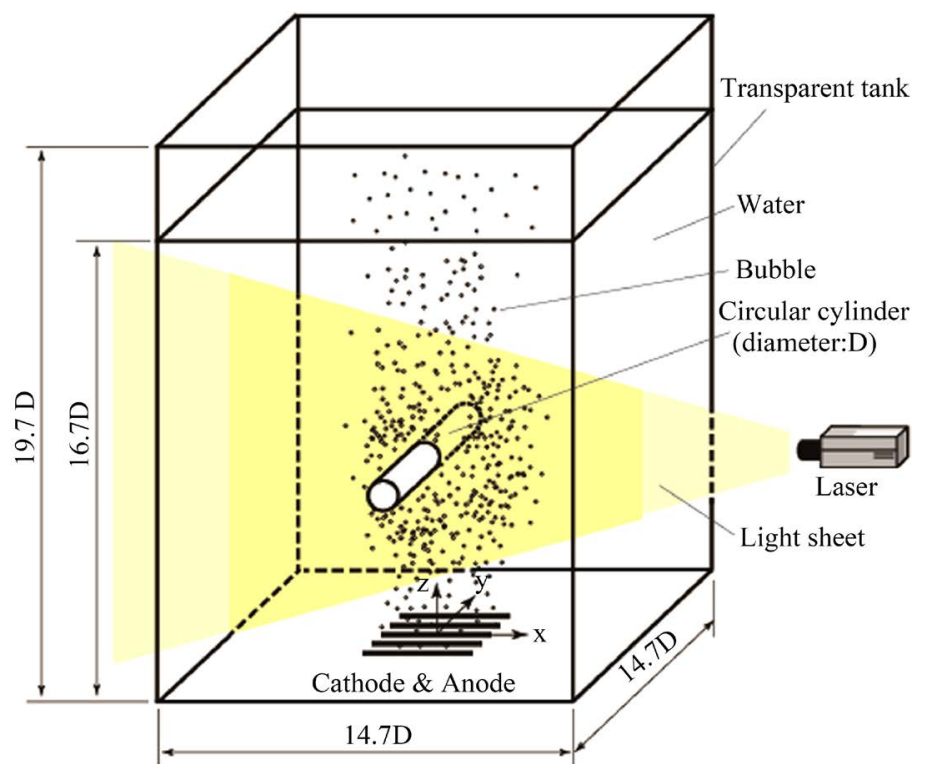

Figure 1. Experimental setup. 


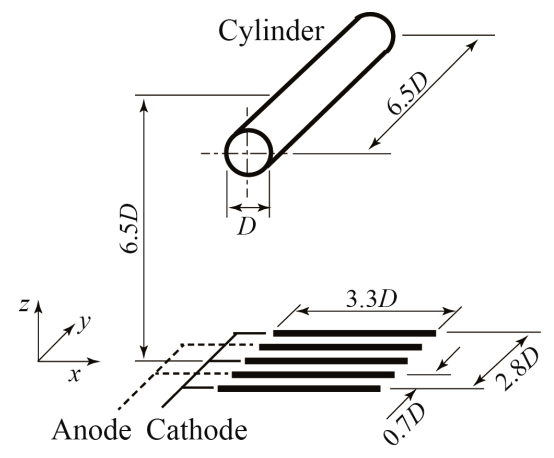

Figure 2. Dimensions and distance of the electrodes and cylinder.

\subsection{Experimental Method and Conditions}

The volume of hydrogen and oxygen bubbles generated per unit time at the carbon rods is controlled by the electric current. In this study, $30 \mathrm{~V}$ is applied, and the experiments are performed using electric current I of 0.1 and $0.3 \mathrm{~A}$. The bubble volume $Q_{g}$ is measured by the downward displacement of water. The $Q_{g}$ values at I $=0.1$ and $0.3 \mathrm{~A}$ are 16 and $53 \mathrm{~mm}^{3} / \mathrm{s}$, respectively.

The bubbles are visualized using a laser light sheet with $0.5 \mathrm{~W}$ power, $532 \mathrm{~nm}$ wavelength, and $1 \mathrm{~mm}$ thickness. The bubble images in the central section ( $x$-z section) perpendicular to the cylinder axis and those in the central section ( $y$-z section) parallel to the cylinder axis are captured using a video camera to investigate the bubble motion.

The bubble diameter is measured in the upper regions of the cylinder using the bubble images acquired at 23 positions in the central section ( $x-z$ section) perpendicular to the cylinder axis. At each position, a small rectangular section is arranged, as shown in Figure 3. The side lengths in the horizontal $(x)$ and vertical $(z)$ directions are $0.53 D$ and $0.33 D$, respectively. The diameter of the bubbles inside each section is measured to calculate the average diameter.

The bubble diameter is smaller than $0.06 \mathrm{~mm}$ at all measurement sections, as discussed below. The bubble velocity in the $x-z$ section is measured with a particle image velocimetry (PIV) system by treating the bubbles as tracer particles. The frame rate and shutter speed of the camera are $200 \mathrm{fps}$ and 1/1000 s, respectively.

The water flow around the cylinder is visualized using fluorescent paint (Rhodamine B). First, the lower-half surface of the cylinder is thinly coated with transparent glue mixed with the fluorescent paint, as shown in Figure 4. The axial length of the coated part is $1.7 \mathrm{D}$. After the glue dries, the cylinder is placed in the water tank. Because the water flow dissolves the paint coated on the cylinder surface, the paint can be used to visualize the water flow. The trajectories of the paint correspond to the water streaklines.

\section{Results and Discussion}

\subsection{Characteristics of the Bubble Plume at Cylinder-Free Condition}

First, the fundamental characteristics of the bubble plume are investigated for this particular experimental setup. The electrolysis of water is started before placing the cylinder in the tank. The bubbles generated at the cathode and anode on the tank bottom rise in the tank due to buoyancy. The bubble plume forms after the bubbles reach the water surface. Figure 5 shows the visualized bubble images at the central vertical section $(x-z$ section) of the tank. The images at $5.5 \leqq z / D \leqq 9$ are presented. In the case of the bubble flow rate of $Q_{g}=16 \mathrm{~mm}^{3} / \mathrm{s}$, a number of bubbles concentrate at $-0.5 \leqq x / D \leqq 0.5$ near the central axis of the tank. The bubble distribution is mostly non-uniform and resembles a belt. The bubbles form cluster locally, especially at the periphery of the bubble plume $(x / D \simeq \mp 0.5)$. This is because the large-scale eddies are induced by the water velocity shear layers at the periphery, and accordingly the bubbles are entrained in the eddies. When $Q_{g}=53 \mathrm{~mm}^{3} / \mathrm{s}$, the horizontal width of the region in which the bubbles are distributed is larger than when $Q_{g}=16 \mathrm{~mm}^{3} / \mathrm{s}$. Bubble entrainment in the large-scale eddies occurs at the periphery of the bubble plume $x / D \simeq \mp 1$, similar to the case of $Q_{g}=16$ $\mathrm{mm}^{3} / \mathrm{s}$. 


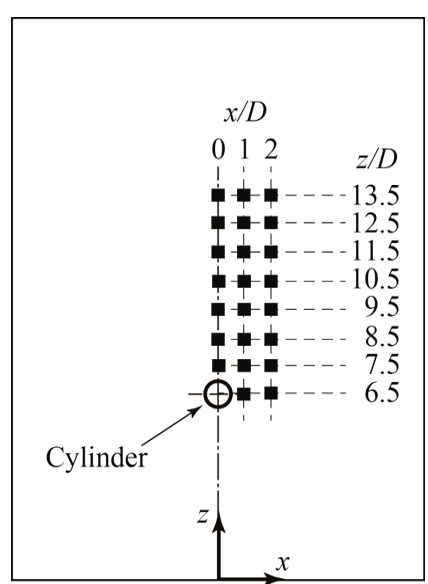

Figure 3. Measurement positions of the bubble diameter.

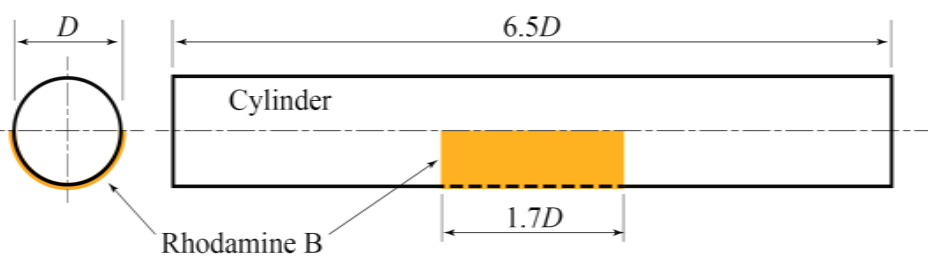

Figure 4. Cylinder partially coated with Rhodamine B.
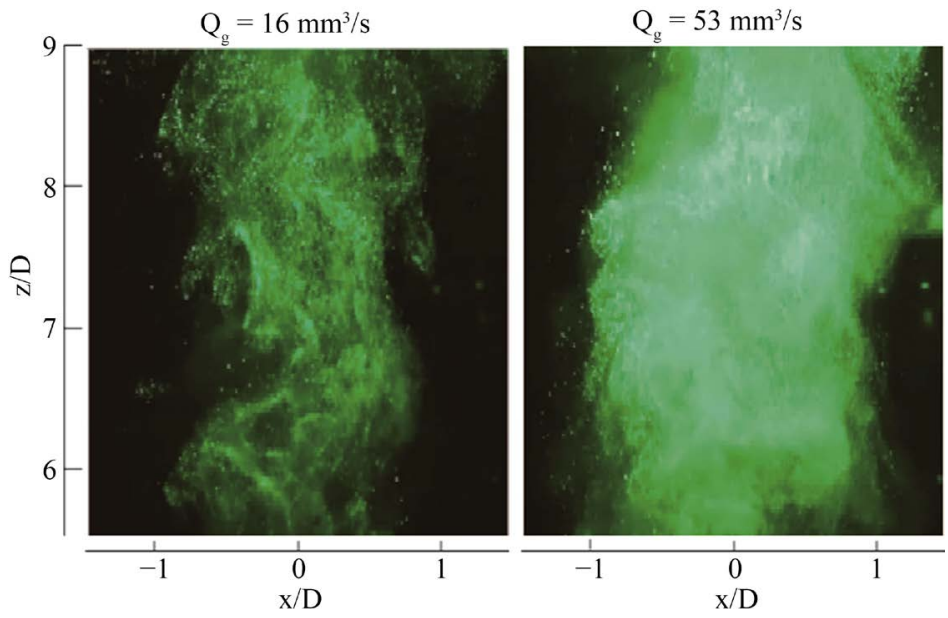

Figure 5. Bubbles in the central vertical cross-section of the tank without the cylinder.

Figure 6 shows the distribution of the bubble diameter $d_{b}$. For $Q_{g}=16 \mathrm{~mm}^{3} / \mathrm{s}$, the variations of $d_{b}$ in the horizontal $(x)$ and vertical $(z)$ directions are not remarkable. There are no data at $z / D \leqq 9.5$ at a horizontal distance of $2 D$ from the cylinder. This is because no bubbles pass through this region. For $Q_{g}=53 \mathrm{~mm}^{3} / \mathrm{s}$, the variation of $d_{b}$ is also small. The average bubble diameters at $Q_{g}$ of 16 and $53 \mathrm{~mm}^{3} / \mathrm{s}$ are $0.043 \mathrm{~mm}$ and $0.047 \mathrm{~mm}$, respectively.

The terminal velocity of a small bubble is given by the following equation [13]:

$$
u_{t}=\frac{g d_{b}^{2} \Delta \rho}{12 \mu}
$$

where $g$ is the gravitational constant, $\Delta \rho$ is the density difference between the bubble and the ambient fluid, and $\mu$ is the viscosity of the ambient fluid. Parkinson et al. [14] measured the terminal velocity of bubbles in water 
and confirmed the validity of Equation (1). $d_{b}$ ranged from 10 to $100 \mu \mathrm{m}$. According to Figure 6, the value of $d_{b}$ at position $(x / D, z / D)=(0,0.5)$, where the circular cylinder would be placed, is $0.045 \mathrm{~mm}$ irrespective of the value of $Q_{g}$. The terminal velocity of the bubble is calculated at $1.7 \mathrm{~mm} / \mathrm{s}$ from Equation (1).

The bubble velocity $\boldsymbol{u}_{g}$ is measured by the PIV system in the central vertical section ( $x$ - $z$ section) of the tank, and the time-averaged velocity $\overline{\boldsymbol{u}}_{g}$ during $60 \mathrm{~s}$ is calculated. Figure 7 shows the distribution of $\overline{\boldsymbol{u}}_{g}$ around the plume center $(-0.75 \leqq x / D \leqq 0.75)$, where the velocity is expressed in a non-dimensional form using the bubble terminal velocity $u_{t}(=1.7 \mathrm{~mm} / \mathrm{s})$. For $Q_{g}=16 \mathrm{~mm}^{3} / \mathrm{s}$, the bubbles flow nearly vertically upward. The magnitude of $\overline{\boldsymbol{u}}_{g}$ is much larger than that of $u_{\mathrm{t}}$, indicating that high-velocity vertical water flow is induced by the rising bubbles. $\overline{\boldsymbol{u}}_{g}$ is maximum at the plume center $(x=0)$ and decreases with increasing horizontal distance $|x|$ from the center. This is attributed to the bubble concentration around the plume center (Figure 5). Note that the velocity variation in the vertically upward direction (z-direction) is small. This suggests that the plume develops spatially in this direction. The velocity for $Q_{g}=53 \mathrm{~mm}^{3} / \mathrm{s}$ is higher than that for $Q_{g}=16 \mathrm{~mm}^{3} / \mathrm{s}$. The decrease with increasing $|x|$ is lower because the bubble distribution is broader in the horizontal direction owing to the larger bubble flow rate (Figure 5), and therefore, the induced water flow has higher velocity in the broader region. The $\overline{\boldsymbol{u}}_{g}$ variation in the vertically upward direction is small, as in the case of $Q_{g}=16 \mathrm{~mm}^{3} / \mathrm{s}$. Because the bubble diameter is smaller than $0.047 \mathrm{~mm}$ (Figure 6), the bubble velocity is assumed to be the same as that of water. Thus, Figure 7 shows the approximate water velocity distribution.

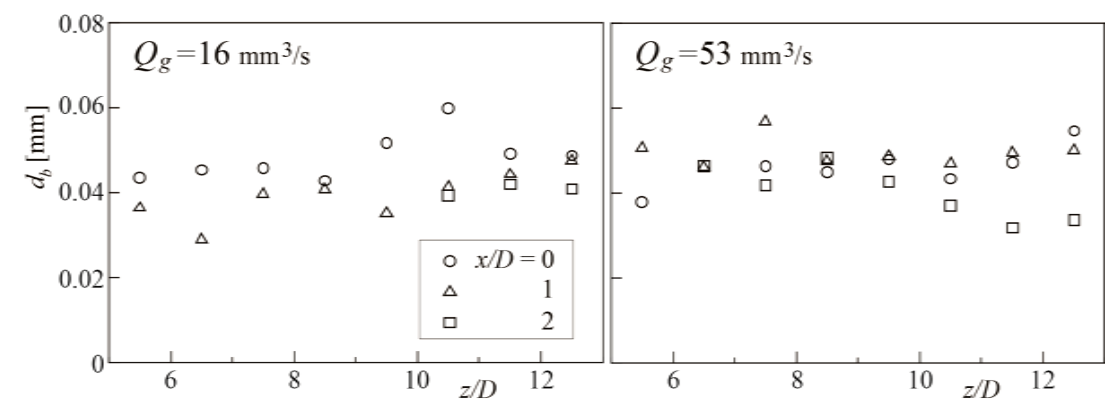

Figure 6. Distribution of the bubble diameter in the vertical cross-section of the tank without the cylinder.

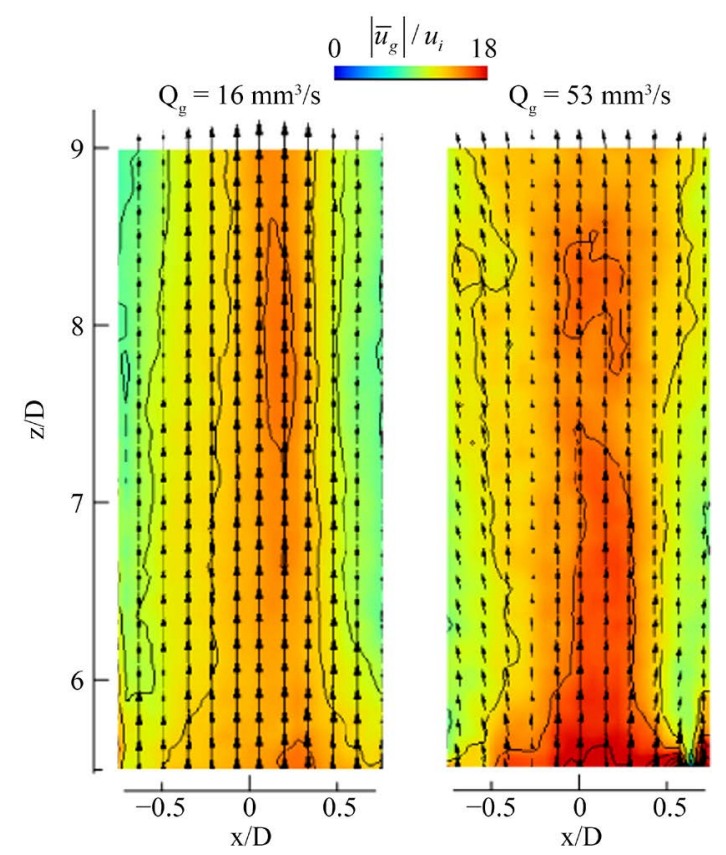

Figure 7. Bubble velocity in the central vertical cross-section of the tank without the cylinder. 


\subsection{Behavior of the Bubble Plume around the Cylinder}

Figure 8 shows images of the bubbles in the central vertical section ( $x$-z section) perpendicular to the cylinder axis. For $Q_{g}=16 \mathrm{~mm}^{3} / \mathrm{s}$, the rising bubbles collide with the front surface of the cylinder and form a layer along the lower-half surface of the cylinder. The bubble layer is separated from both sides of the cylinder $(x / D=\mp 0.5$, $z / D=6.5$ ) and the separated bubbles flow almost vertically. This is attributed to the separation and convection of water shear layers, as discussed below. The separated layers of the bubbles roll up downstream of the cylinder. The region where bubbles are hardly entrained is just behind the cylinder. For $Q_{g}=53 \mathrm{~mm}^{3} / \mathrm{s}$, the separated layers are observed at the cylinder sides. They convect more vertically when compared with the results for $Q_{g}=16$ $\mathrm{mm}^{3} / \mathrm{s}$. Presumably, the rising velocity of the plume increases.

The bubble distribution in the vertical section ( $y$-z section) passing through the cylinder axis is shown in Figure 9. Irrespective of the $Q_{g}$ value, the bubbles colliding with the cylinder move in the horizontal $(y)$ direction along the lower-half surface of the cylinder. Thus, the bubble distribution is broader in the horizontal direction downstream of the cylinder. Bubble clusters appear at the plume edges downstream of the cylinder, and a bubble plume with three-dimensional features is seen.

Figure 10 shows the bubbles and Rhodamine B around the cylinder for $Q_{g}=16 \mathrm{~mm}^{3} / \mathrm{sat}$ every $1 / 3 \mathrm{~s}$ after the reference time $t_{0}$. The Rhodamine B images show that the water separated shear layers originating at both sides

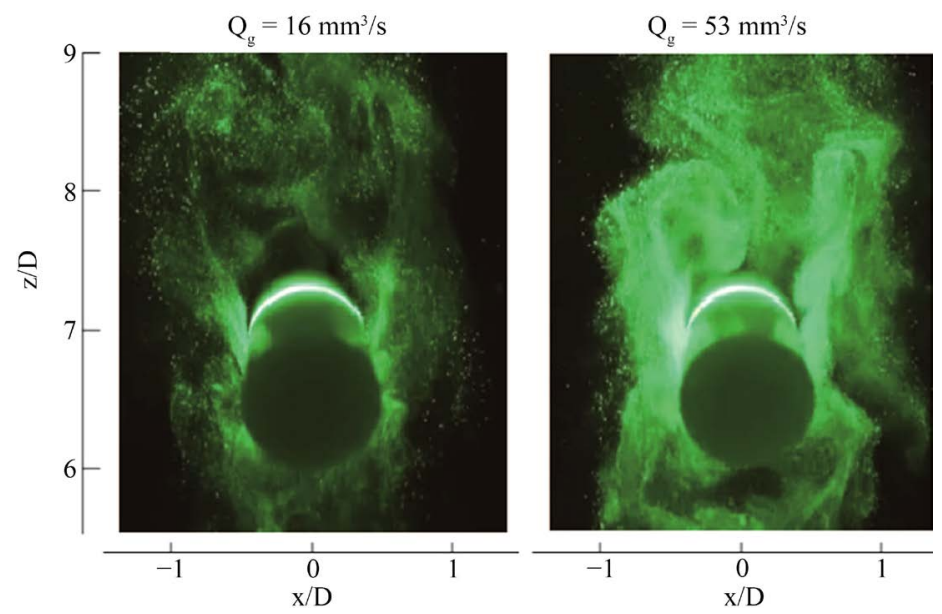

Figure 8. Bubbles in the central cross-section perpendicular to the cylinder axis.
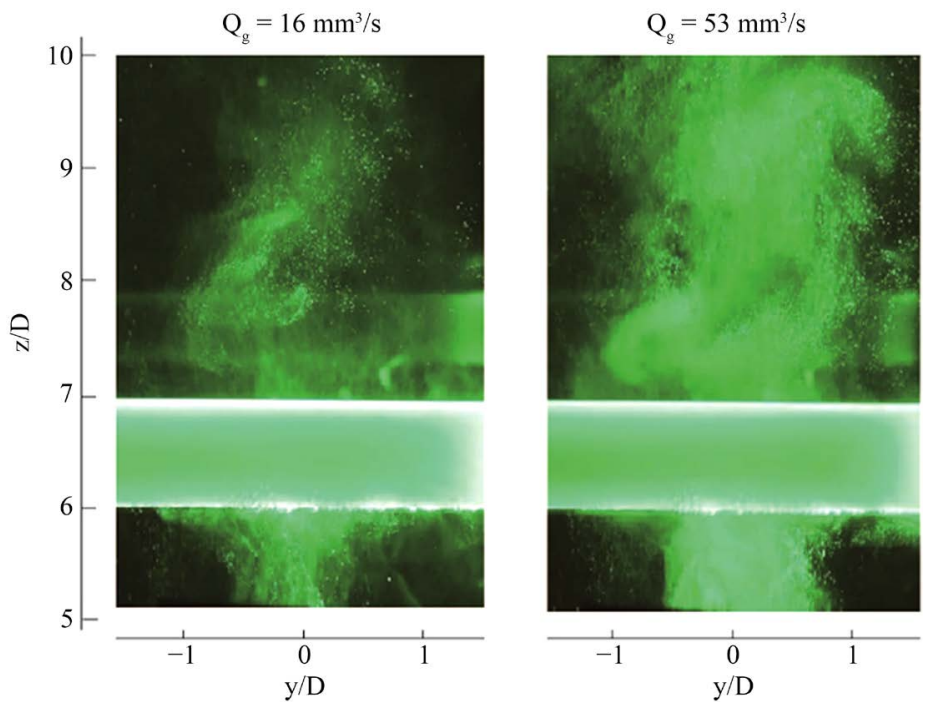

Figure 9. Bubbles in the cross-section passing through the cylinder axis. 
of the cylinder convect with rolling up. Several bubbles are distributed along the shear layers. The water and bubbles separate at the cylinder surface and convect together. Large-scale eddies induced by the roll-up of the separated shear layers are observed downstream of the cylinder, and some bubbles are entrained in the eddies.

Bubbles and Rhodamine B images at $Q_{g}=53 \mathrm{~mm}^{3} / \mathrm{s}$ are shown in Figure 11. In comparison with the images at $Q_{g}=16 \mathrm{~mm}^{3} / \mathrm{s}$, the water separated shear layers convect more vertically and the position where the roll-up occurs shifts downstream.

The bubble diameter $d_{b}$ distributes as shown in Figure 12. Compared with the result for the cylinder-free condition in Figure 6, no effect of the cylinder on $d_{b}$ is observed. The average diameters at $Q_{g}=16$ and 53 $\mathrm{mm}^{3} / \mathrm{s}$ are 0.047 and $0.045 \mathrm{~mm}$, respectively.

The distribution of the time-averaged bubble velocity $\overline{\mathbf{u}}_{g}$ in the central vertical section ( $x-z$ section) is shown in Figure 13, where the distribution of the same region as in Figure 7 is presented. At $Q_{g}=16 \mathrm{~mm}^{3} / \mathrm{s}$, the velocity decreases along the plume centerline while approaching the leading point of the cylinder. A stagnant flow is seen at the leading point. The bubbles colliding with the leading point flow along the cylinder surface with being accelerated, and their velocity reaches the maximum at the cylinder sides. This is attributed to the fact that the water is accelerated owing to the curvature of the streamline along the cylinder surface. The separated layers
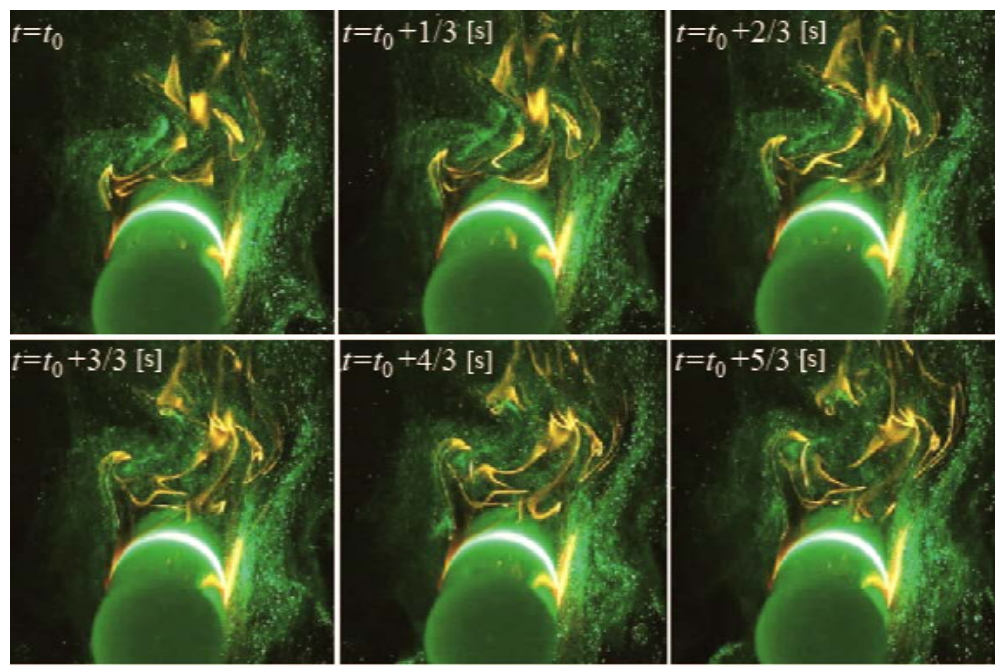

Figure 10. Time-variations of bubbles and Rhodamine B in the central vertical cross-section perpendicular to the cylinder axis $\left(Q_{g}=16 \mathrm{~mm}^{3} / \mathrm{s}\right)$.
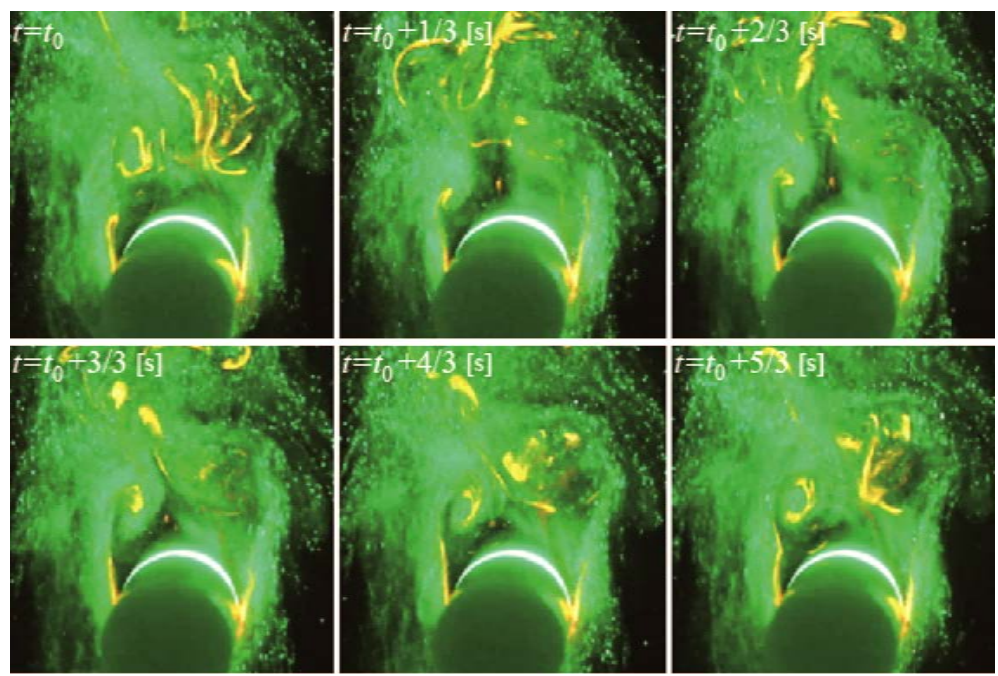

Figure 11. Time-variations of bubbles and Rhodamine B in the central vertical cross-section perpendicular to the cylinder axis $\left(Q_{g}=53 \mathrm{~mm}^{3} / \mathrm{s}\right)$. 


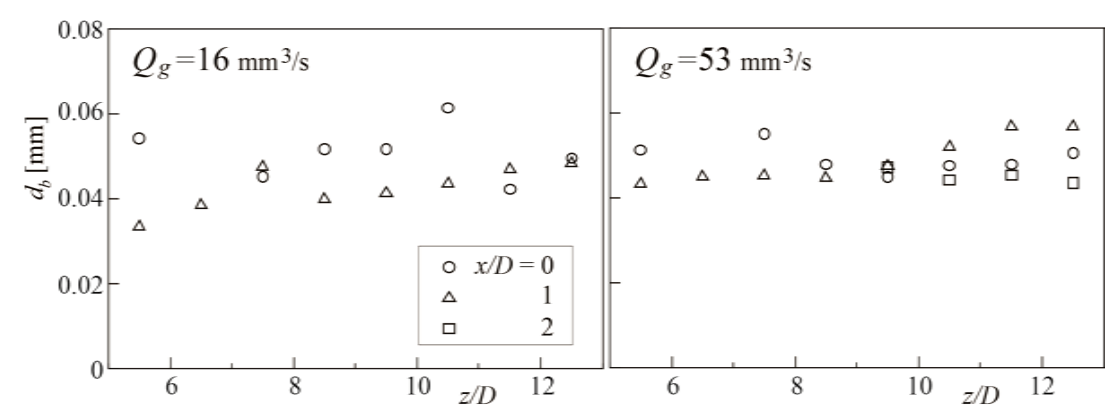

Figure 12. Bubble diameter in the central cross-section perpendicular to the cylinder axis.

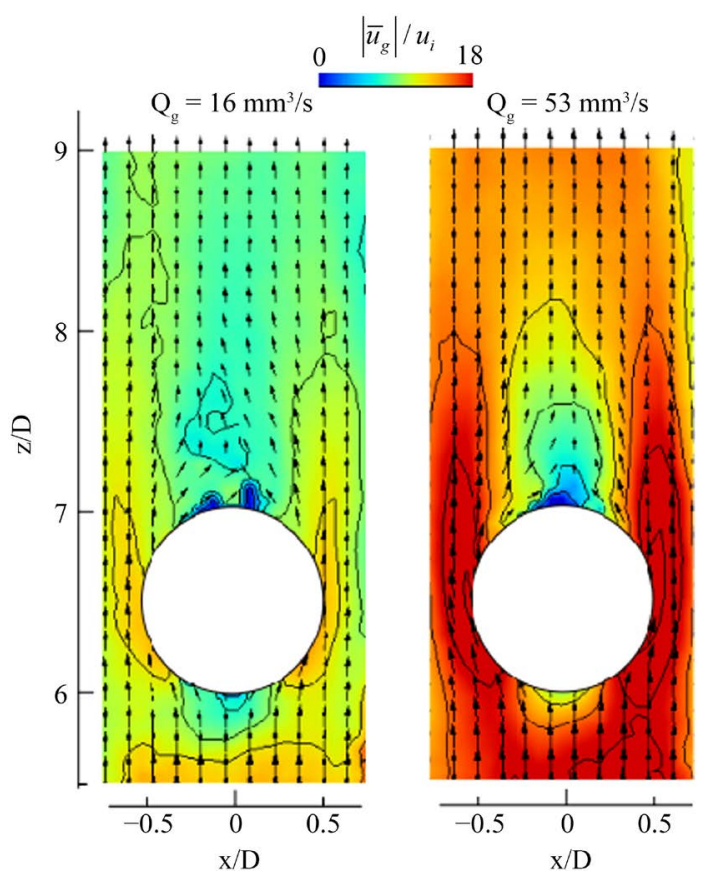

Figure 13. Bubble velocity in the central cross-section perpendicular to the cylinder axis.

originating at both sides of the cylinder are reconfirmed. Note that the velocity is lower near the central region just behind the cylinder and the wake ranges in the vertical direction. At $Q_{g}=53 \mathrm{~mm}^{3} / \mathrm{s}$, the velocity distribution is similar to that at $Q_{g}=16 \mathrm{~mm}^{3} / \mathrm{s}$. Nevertheless, the wake region is narrower and the velocity recovers promptly. On the cross-section at $z / D=5$, upstream of the cylinder, the averaged velocity $u_{g 0}$ is calculated. The values of $u_{g 0}$ at $Q_{g}=16$ and $53 \mathrm{~mm}^{3} / \mathrm{s}$ are 23.4 and $32.7 \mathrm{~mm} / \mathrm{s}$, respectively.

\subsection{Periodic Flow Induced by Cylinder}

The horizontal bubble velocity $u_{g x}$ is measured at a position in the central vertical section ( $x-z$ section) perpendicular to the cylinder axis. The coordinates of the position $(x, z)$ are $(0.63 D, 7.7 D)$. The luminance of the bubble $L$ is assumed to be proportional to the bubble volume fraction and is measured at $(1.3 D, 7.7 D)$. Figure 14 shows the two positions. When measuring $L$, a square region of $11 \times 11$ pixels is set around the measurement position to obtain the luminance at 121 pixels by the 256 gradations and calculate the average.

Figure 15 shows the time variation of $u_{g x}$ at $Q_{g}=16 \mathrm{~mm}^{3} / \mathrm{s}$. When the cylinder is not placed, no marked variations are seen. However, variations with almost constant period and amplitude appear when the cylinder is placed. The cylinder induces a bubbly flow with periodic oscillation in the horizontal direction. The velocity is measured at the location where the water separated shear layer with bubbles passes periodically (Figure 10). 


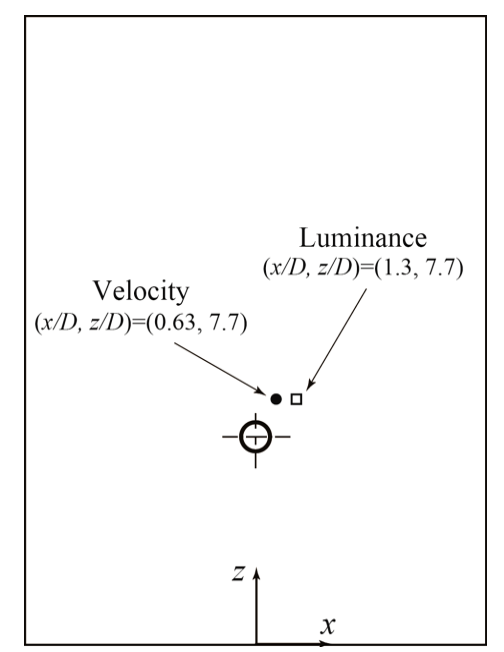

Figure 14. Measurement positions of the bubble velocity and luminance in the central cross-section perpendicular to the cylinder axis.

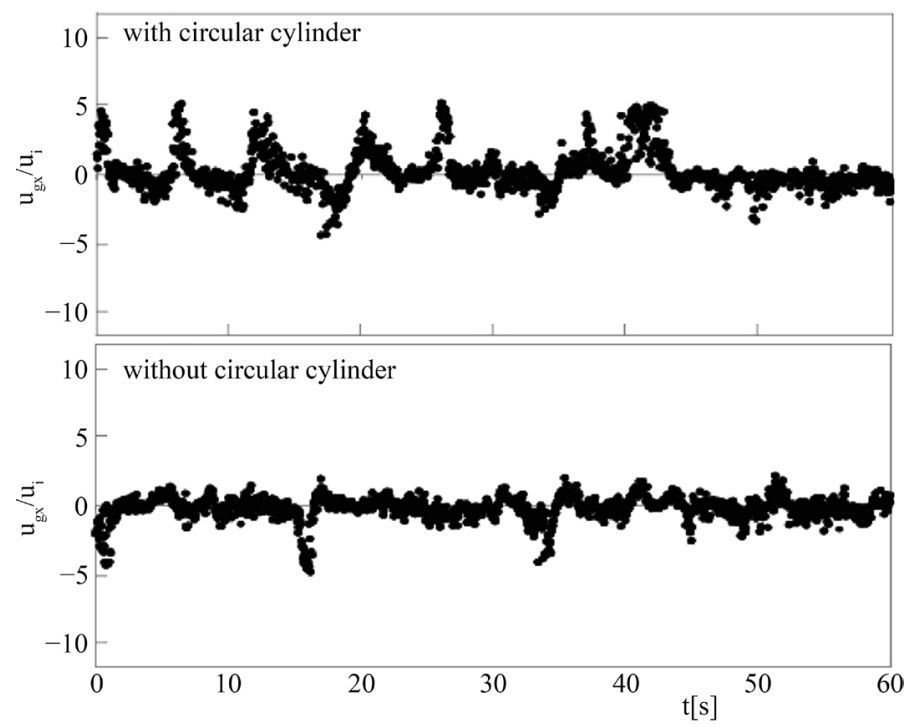

Figure 15. Time-variation of the horizontal component of the bubble velocity for $Q_{g}=16 \mathrm{~mm}^{3} / \mathrm{s}$.

The time variation of $u_{g x}$ at $Q_{g}=53 \mathrm{~mm}^{3} / \mathrm{s}$ is shown in Figure 16. Though $u_{g x}$ varies even in the cylinder-free condition, the variation induced by the cylinder is nearly periodic and the amplitude increases. The amplitude is larger than that at $Q_{g}=16 \mathrm{~mm}^{3} / \mathrm{s}$ because of the higher velocity of the separated layers.

The frequency analysis of $u_{g x}$ shows the dominant frequency $f_{B}$, which is 0.14 and $0.11 \mathrm{~Hz}$ at $Q_{g}=16$ and 53 $\mathrm{mm}^{3} / \mathrm{s}$, respectively. The non-dimensional frequency $f_{B} D / u_{\mathrm{g} 0}$ is calculated, where $u_{g 0}$ is the velocity at $z / D=5$ upstream of the cylinder. The values at $Q_{g}=16$ and $53 \mathrm{~mm}^{3} / \mathrm{s}$ are 0.18 and 0.1 , respectively. The Reynolds number of the bubble plume is defined as $\operatorname{Re}=u_{g 0} D / v$, where $v$ is the kinematic viscosity of water. The Re values at $Q_{g}=16$ and $53 \mathrm{~mm}^{3} / \mathrm{s}$ are 702 and 981 , respectively.

The vortex shedding frequency $f$ behind a circular cylinder for uniform flow with velocity $U$ is expressed by the Strouhal number St $(=f D / U)$. Figure 17 shows the relation between Re $(=U D / v)$ and Stinvestigated by Roshko [15]. The solid line in the figure is the Roshko's correlation, which is given as

$$
\mathrm{St}=0.212-2.7 / \mathrm{Re}
$$

The values of $S t=f_{B} D / u_{g 0}$ are also shown by painted symbols in Figure 17. They are much lower than the 


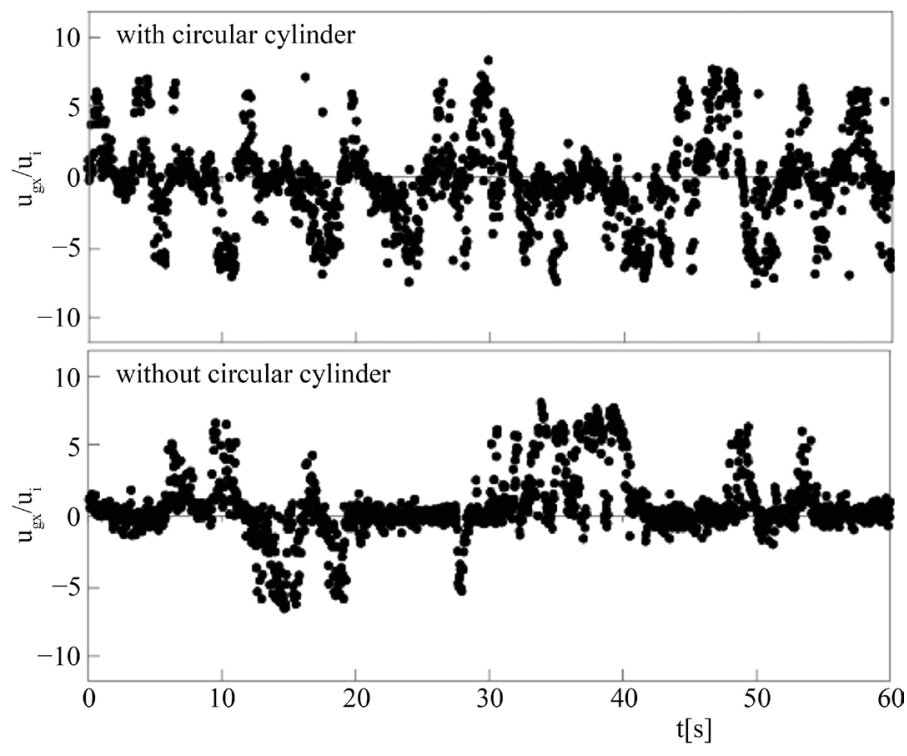

Figure 16. Time-variation of the horizontal component of the bubble velocity for $Q_{g}=53 \mathrm{~mm}^{3} / \mathrm{s}$.

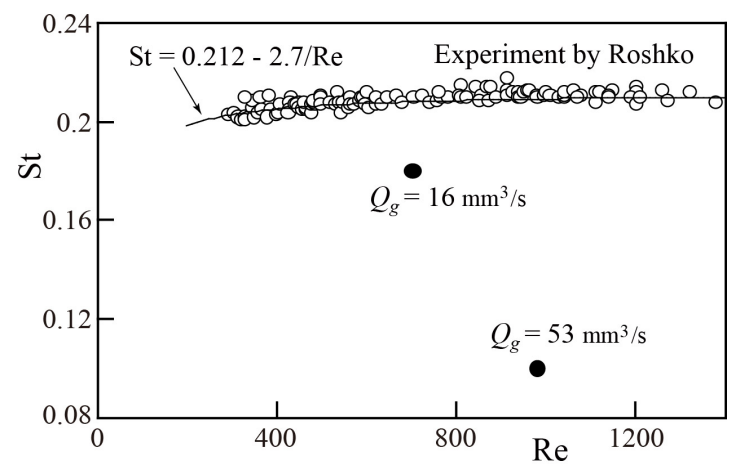

Figure 17. Dependence of Strouhal number on Reynolds number.

results for uniform flow. This may be attributed to the non-uniform distribution of the fluid density due to the bubbles.

Figure 18 shows the time variation of $L$ at $Q_{g}=53 \mathrm{~mm}^{3} / \mathrm{s}$, where $L$ is normalized by the maximum value (255). $L$ varies irregularly in the cylinder-free condition. Nevertheless, a regular variation is produced when the cylinder is placed. The time-averaged $L$ and amplitude increase. The variation of $L$ also demonstrates the periodicity of the water separated shear layer with bubbles.

The frequency analysis of $L$ shows that the dominant frequency $f_{L}$ is $0.14 \mathrm{~Hz}$. This is slightly larger than that of $u_{g x}(0.11 \mathrm{~Hz})$. Apparently, the positions where $L$ and $u_{g x}$ are minimum or maximum do not always correspond.

\section{Conclusions}

In a water tank, a cylinder with diameter $30 \mathrm{~mm}$ is placed inside a bubble plume induced by rising gas bubbles with diameter smaller than $0.06 \mathrm{~mm}$. The bubbles and water flow around the cylinder are visualized, and the bubble velocity distribution is measured. The following can be concluded.

1) Bubbles colliding with the front surface of the cylinder are distributed along the lower-half surface of the cylinder. They separate at both sides of the cylinder, and the separated bubble layers convect almost vertically. The bubbles are entrained around the plume centerline downstream of the cylinder. The bubbles hardly distribute just behind the cylinder.

2) The water shear layers also separate at both sides of the cylinder and convect with rolling up. The water 


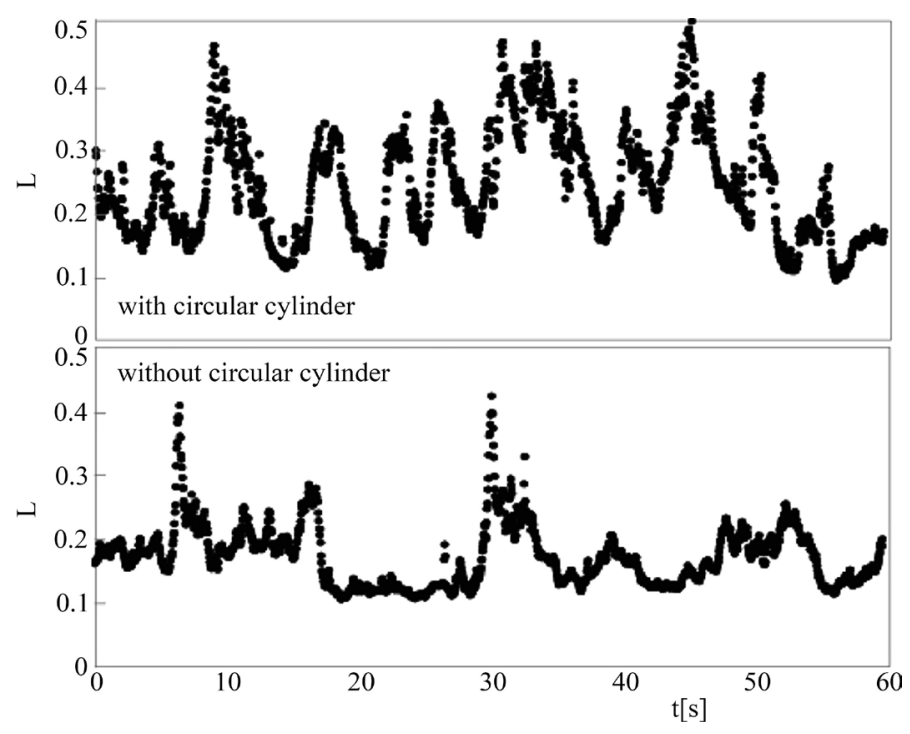

Figure 18. Time-variation of luminance for $Q_{g}=53 \mathrm{~mm}^{3} / \mathrm{s}$.

and bubbles separate and flow together. The bubble entrainment downstream of the cylinder is caused by the large-scale eddies produced by the roll-up of the water shear layers.

3) In the central vertical section perpendicular to the cylinder axis, a stagnant flow of bubbles is seen at the leading point of the cylinder. The bubbles collide with the front surface of the cylinder flow with being accelerated along the cylinder surface, and their velocity is maximum at the sides of the cylinder. A wake region of extremely low velocity exists just behind the cylinder.

4) The cylinder induces periodic flow behind it. When the dominant frequency of the horizontal velocity of the bubble is expressed in a non-dimensional for musing the bubble velocity upstream of the cylinder, it is smaller than the vortex shedding frequency behind a cylinder for uniform flow.

\section{References}

[1] Hussain, N.A. and Siegel, R. (1976) Liquid Jet Pumped by Rising Gas Bubbles. Journal of Fluids Engineering, 98, 49-56. http://dx.doi.org/10.1115/1.3448206

[2] Leitch, A.M. and Baines, W.D. (1989) Liquid Volume Flux in a Weak Bubble Plume. Journal of Fluid Mechanics, 205, 77-98. http://dx.doi.org/10.1017/S0022112089001953

[3] Milgram, J.H. (1983) Mean Flow in Round Bubble Plumes. Journal of Fluid Mechanics, 133, 345-376. http://dx.doi.org/10.1017/S0022112083001950

[4] Socolofsky, S.A. and Adams, E.E. (2005) Role of Slip Velocity in the Behavior of Stratified Multiphase Plumes. Journal of Hydraulic Engineering, 131, 273-282. http://dx.doi.org/10.1061/(ASCE)0733-9429(2005)131:4(273)

[5] Alam, M. and Arakeri, V.H. (1993) Observations on Transition in Plane Bubble Plumes. Journal of Fluid Mechanics, 254, 363-374. http://dx.doi.org/10.1017/S0022112093002174

[6] Murai, Y. and Matsumoto, Y. (1997) Numerical Analysis of Detailed Flow Structures in Bubble Plume, 2nd Report, Three-Dimensional Flow Structure and Turbulence Mechanism. Transactions of the Japan Society of Mechanical Engineers Series B, 63, 2283-2288.

[7] Uchiyama, T. and Matsumura, S. (2010) Three-Dimensional Vortex Method for the Simulation of Bubbly Flow. Journal of Fluids Engineering, 132, 101402. http://dx.doi.org/10.1115/1.4002574

[8] Wu, J.K. (1994) Two Problems of Computer Mechanics Program System. Proceedings of Finite Element Analysis and $C A D$, Peking University Press, Beijing, 9-15.

[9] Chen, B., Wang, Z. and Uchiyama, T. (2014) Numerical Simulation of Bubble Cluster Induced Flow by Three-Dimensional Vortex-in-Cell Method. Trans. Journal of Fluids Engineering, 136, 081301. http://dx.doi.org/10.1115/1.4026968

[10] Uchiyama, T., Yoshii, Y., Chen, B. and Wang, Z. (2015) Numerical Simulation of Bubbly Flow by an Improved Vortex in Cell Method. Advances and Applications in Fluid Mechanics, 17, 91-114.

http://dx.doi.org/10.17654/15-AAFM-01701-091 
[11] Uchiyama, T. (2000) Numerical Analysis of Air-Water Two-Phase Flow across a Staggered Tube Bundle Using an Incompressible Two-Fluid Model. Nuclear Science and Engineering, 134, 281-292.

[12] Murai, Y., Sasaki, T., Ishikawa, M. and Yamamoto, F. (2005) Bubble-Driven Convection around Cylinders Confined in a Channel. Journal of Fluids Engineering, 127, 117-123. http://dx.doi.org/10.1115/1.1852478

[13] Levich, V.G. (1962) Physicochemical Hydrodynamics. Prentice-Hall, Englewood Cliffs.

[14] Parkinson, L., Sedev, R., Fornasiero, D. and Ralston, J. (2008) The Terminal Rise Velocity of 10 - $100 \mu \mathrm{m}$ Diameter Bubbles in Water. Journal of Colloid and Interface Science, 322, 168-172. http://dx.doi.org/10.1016/j.jcis.2008.02.072

[15] Roshko, A. (1954) On the Development of Turbulent Wakes from Vortex Streets. NACA Report 1191, National Advisory Committee for Aeronautics, Washington DC.

\section{Submit or recommend next manuscript to SCIRP and we will provide best service for you:}

Accepting pre-submission inquiries through Email, Facebook, Linkedin, Twitter, etc A wide selection of journals (inclusive of 9 subjects, more than 200 journals)

Providing a 24-hour high-quality service

User-friendly online submission system

Fair and swift peer-review system

Efficient typesetting and proofreading procedure

Display of the result of downloads and visits, as well as the number of cited articles

Maximum dissemination of your research work

Submit your manuscript at: http://papersubmission.scirp.org/ 